\title{
Bird Diversity in Riverscapes of Ayodhya District, Uttar Pradesh
}

\author{
Yashmita-Ulman \\ Department of Silviculture \& Agroforestry, College of Horticulture \& Forestry \\ ANDUAT, Kumarganj, Ayodhya-224 229, India \\ E-mail: yashmita2018@gmail.com
}

\begin{abstract}
The bird diversity using line transect method along the $45 \mathrm{~km}$ stretch of Saryu river between Aharauli and Pitapur in eastern Uttar Pradesh was assessed. A total of sixty eight bird species, belonging to 56 genera, 35 families and 12 orders were recorded from the study area. Passeriformes (24) had the maximum number of bird species. The maximum encounter rate was for Indian Silverbill (2.685) and the minimum for Blue-tailed Bee-eater (0.054). Out of 68 bird species recorded, 56 bird species (82\%) were resident, 11 bird species (16\%) were winter visitors and only 1 bird species (1\%) was summer visitor. A large number of Carnivores (29 species, $43 \%)$ were recorded in the area. Of the recorded species, nine were in the threatened category of IUCN Red List and eighteen species $(26 \%)$ of birds had a declining population trend globally highlighting the conservation value of riverscapes of Saryu river. This study has helped in generating a baseline information on the bird diversity of the Saryu river and emphasis on carrying out long-term monitoring of birds in such threatened ecosystem.
\end{abstract}

Keywords: Avian, Freshwater ecosystems, Feeding guild, Ayodhya, Saryu river

Freshwater is a natural resource which is important in terms of economy, culture, science and education (Dudgeon et al 2006). Freshwater habitats including rivers, streams, lakes, oxbows, wetlands and estuaries etc. and their adjacent areas called riparian zones alone support around $6 \%$ of world known species (Hawksworth and Kalin-Arroyo 1995). Birds are an important constituent of the riverscape. A riverscape is a landscape constituting of the streams, floodplains and riparian zones along a river channel (Wiens 2002). The riverscapes provide essential dispersal pathways, shelter, breeding and nesting sites for the birds, thus supporting high bird species diversity (Sinha et al 2019). But as these riverscapes are exposed to anthropogenic pressure due to its productivity and developmental activities, these areas are the most threatened ecosystems of the world (Revenga et al 2005). Thus, leading to loss of the ecosystem and their associated bird diversity. These riverscapes are meagerly managed from conservation point of view. In addition, the existing knowledge on bird diversity in these ecosystems is incomplete and insufficient to assess the impacts of anthropogenic pressures on bird diversity loss.

The river Ganga is one of the largest rivers with many tributaries flowing through $2510 \mathrm{~km}$ from north to east India (Adel 2001). This river supports $8 \%$ of the world's population living in its catchment (Newby 1998). Understanding the bird diversity, abundance and habitat preferences are necessary baseline parameters for planning conservation activities, but such information is meagre from certain tributaries of Ganga. Studies have been conducted on the faunal diversity of some segments along the stretch of Ganga (Behera 1995, Bashir et al 2012). Ghaghra river is one of the principal tributaries of river Ganga. The Ghaghra joins its sub-tributary, the Sharda river and flows through the district of Ayodhya where it is locally known as Saryu river (Dhar and Nandargi 2002, Singh et al 2016). There have been no bird diversity studies done on the Saryu river flowing through Ayodhya district, Uttar Pradesh. Therefore, in this study, an attempt was made to document the bird diversity in the catchment area of river Saryu.

\section{MATERIAL AND METHODS}

The study was conducted in a $45 \mathrm{~km}$ stretch of Saryu river flowing between Aharauli $26.858^{\circ} \mathrm{N}$ and $81.814^{\circ} \mathrm{E}$ and Pitapur $26.782^{\circ} \mathrm{N}$ and $82.256^{\circ} \mathrm{E}$ in Ayodhya district, Uttar Pradesh (Fig. 1). The banks of this river are sandy or muddy; the floodplains are mosaic of agriculture, orchards, forests, grasslands etc. The climate of the district is tropical monsoon. The average temperature varies from $32^{\circ} \mathrm{C}$ in summers to $16^{\circ} \mathrm{C}$ in winters and the average annual rainfall is $1067 \mathrm{~mm}$ (Anonymous 2021). There are three distinct seasons - summer (March to June), rainy (July to October) and winter (November to February). Hundreds of pilgrims visit the river banks of Saryu during Hindu festivals to perform religious ceremonies.

The entire $45 \mathrm{~km}$ stretch of the Saryu river in Ayodhya district was divided into nine segments of $5 \mathrm{~km}$ each. Bird survey was done using line transect method (Gregory et al 2004) in five segments (selected based on their accessibility and feasibility of sampling), so in all covering a stretch of 25 $\mathrm{km}$. Five transects ( $5 \mathrm{~km}$ each) were laid parallel to the river 
and without any overlap in the selected $25 \mathrm{~km}$ segment of river. The bird sampling was done from October 2020 to May 2021 , each month to record birds in winter and summer season. Two replications of each transect were made in both the seasons, making a total field effort of $400 \mathrm{~km}$ for the study. The transect was walked on foot at a speed of 1 to $2 \mathrm{~km} / \mathrm{hr}$ from early morning (30 minutes after sunrise to $11 \mathrm{am}$ ). For each direct sighting, the GPS location, time and habitat were recorded. A standard field guide was used for identification (Grimmett et al 2011). Every species recorded was assigned the taxonomic position, common and scientific names by referring to Praveen et al (2020). The assessment of threat status of the recorded bird species was based on IUCN Red List (2021). Torre-Cuadros et al (2007) was followed to calculate the relative diversity of bird families. The formula is given below:

$$
\mathrm{RDi}=\frac{\text { Number of bird species in a family }}{\text { Total number of species }} \times 100
$$

The sightings obtained from each transect walk was pooled to report encounter rates of species for the entire river stretch. The encounter rate for each species was calculated using the formula 'number detected per km' (Datta and Goyal 2008).

\section{RESULTS AND DISCUSSION}

A total of 68 bird species belonging to 56 genera, 35 families and 12 orders were recorded from the study area (Table 1). Similar studies in riverine areas of Ganga indicate that the present study has yielded fewer species of birds in contrast to 86 bird species reported by Behera (1995), but higher species of birds when compared to study done by Bashir et al (2012) which yielded 55 bird species. The high bird diversity in the riverscape may be attributed to the mosaic nature of the area which consists of agricultural fields, mango and guava orchards, marshlands, forest patches which may have provided diverse habitat to meet the different requirements of birds. Amongst the order, Passeriformes (24) had the maximum number of species, followed by Pelecaniformes (11) (Fig. 2). The order of Caprimulgiformes, Galliformes and Psittaciformes (1 each) had the lowest number of species (Fig. 2). The order of Passeriformes (13) had the maximum number of families, followed by Charadriiformes (6) (Fig. 2). The family Ardeidae (6) had the highest number of species, followed by Accipitridae, Columbidae, Motacillidae and Sturnidae (4 each) (Table 2). In India, Passeriformes are known to be the most dominant order (Praveen et al 2016).

The maximum encounter rate was for Indian Silverbill (Euodice malabarica) (2.685) and the minimum for Bluetailed Bee-eater (Merops philippinus) (0.054) (Table 1). This result is dissimilar to the result of Bashir et al (2012) who have reported highest encounter rate of Little Cormorant and the least of Indian White-rumped vulture. Out of 68 bird species recorded, 56 bird species (82\%) were resident, 11 bird species $(16 \%)$ were winter visitors and only 1 bird species (1\%) was summer visitor (Fig. 3). The study area is a part of

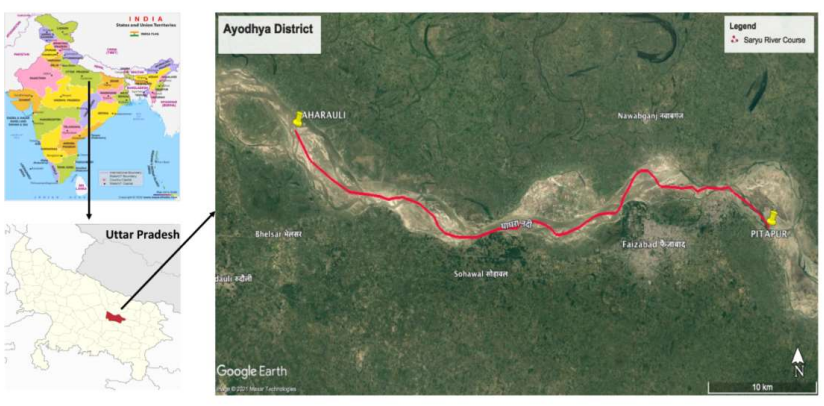

Fig. 1. Location map of study area

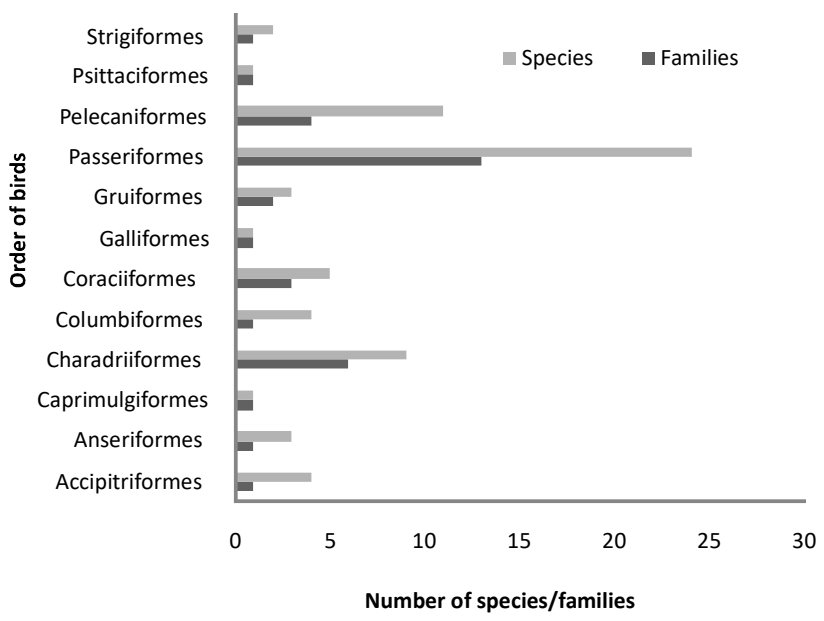

Fig. 2. Order wise bird community composition in riverscapes of Ayodhya district, Uttar Pradesh

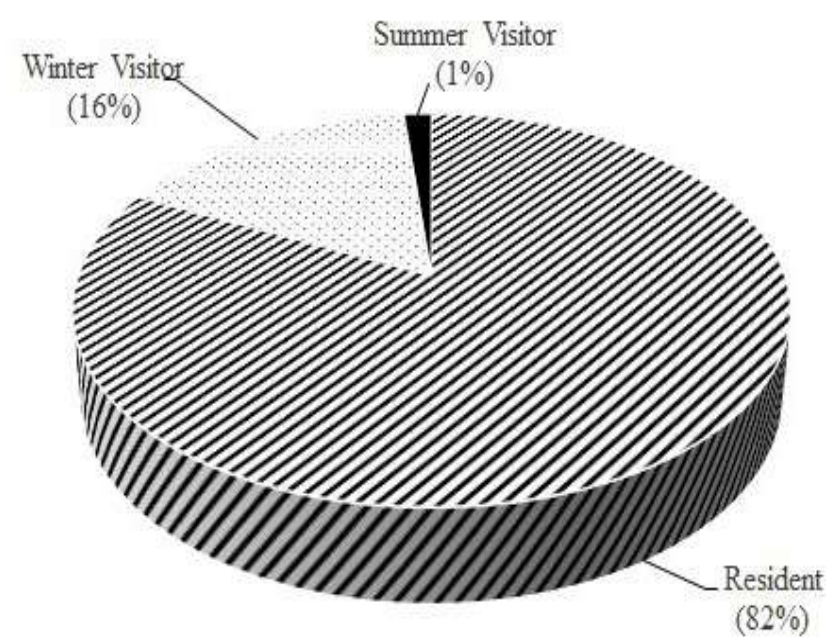

Fig. 3. Seasonal status of bird species in riverscapes of Ayodhya district, Uttar Pradesh 
Table 1. Checklist and encounter rate of avifauna recorded in riverine areas of Ayodhya district, Uttar Pradesh, India

\begin{tabular}{|c|c|c|c|c|c|c|c|c|c|}
\hline \multirow{2}{*}{$\begin{array}{l}\text { Order/Family/ } \\
\text { Common name }\end{array}$} & \multirow[t]{2}{*}{ Scientific name } & \multirow{2}{*}{$\begin{array}{l}\text { Residential } \\
\text { status }\end{array}$} & \multirow{2}{*}{$\begin{array}{l}\text { Feeding } \\
\text { guild }\end{array}$} & \multicolumn{4}{|c|}{ Conservation status } & \multirow[t]{2}{*}{ Habitat (s) } & \multirow{2}{*}{$\begin{array}{l}\text { Encounter } \\
\text { rate }\end{array}$} \\
\hline & & & & $\begin{array}{l}\text { IUCN list } \\
(2021)\end{array}$ & $\begin{array}{l}\text { CITES } \\
(2012)\end{array}$ & $\begin{array}{l}\text { IWPA } \\
(1972)\end{array}$ & $\begin{array}{l}\text { Global } \\
\text { status }\end{array}$ & & \\
\hline \multicolumn{10}{|c|}{ Accipitriformes Accipitridae (4) } \\
\hline Black Kite & $\begin{array}{l}\text { Milvus migrans } \\
\text { (Boddaert, 1783) }\end{array}$ & $\mathrm{R}$ & C & LC & II & I & $\rightarrow$ & $R B, A, F, M$ & 0.21 \\
\hline Black-winged Kite & $\begin{array}{l}\text { Elanus caeruleus } \\
\text { (Desfontaines, 1789) }\end{array}$ & $\mathrm{R}$ & C & LC & II & I & $\rightarrow$ & A & 0.092 \\
\hline Egyptian Vulture & $\begin{array}{l}\text { Neophron } \\
\text { percnopterus } \\
\text { (Linnaeus, 1758) }\end{array}$ & $\mathrm{R}$ & $\mathrm{C}$ & EN & - & I & $\downarrow$ & $A, M$ & 0.082 \\
\hline Shikra & $\begin{array}{l}\text { Accipiter badius } \\
\text { (Gmelin, 1788) }\end{array}$ & $\mathrm{R}$ & C & LC & II & I & $\rightarrow$ & $A, F$ & 0.338 \\
\hline \multicolumn{10}{|c|}{ Anseriformes Anatidae (3) } \\
\hline Common Pochard & $\begin{array}{l}\text { Aythya ferina } \\
\text { (Linnaeus, 1758) }\end{array}$ & WV & $\mathrm{O}$ & VU & - & IV & $\downarrow$ & $\mathrm{RC}, \mathrm{M}$ & 0.432 \\
\hline $\begin{array}{l}\text { Indian Spot-billed } \\
\text { Duck }\end{array}$ & $\begin{array}{l}\text { Anas poecilorhyncha } \\
\text { (Forster, 1781) }\end{array}$ & $\mathrm{R}$ & $\mathrm{O}$ & LC & - & IV & $\downarrow$ & $\mathrm{RC}, \mathrm{M}$ & 0.974 \\
\hline Ruddy Shelduck & $\begin{array}{l}\text { Tadorna ferruginea } \\
\text { (Pallas, 1764) }\end{array}$ & WV & $\mathrm{O}$ & LC & - & IV & $?$ & $\mathrm{RC}$ & 0.56 \\
\hline \multicolumn{10}{|c|}{ Caprimulgiformes Upupidae (1) } \\
\hline Common Hoopoe & $\begin{array}{l}\text { Upupa epops } \\
\text { (Linnaeus, 1758) }\end{array}$ & $\mathrm{R}$ & $\mathrm{O}$ & LC & - & IV & $\downarrow$ & $\mathrm{RB}, \mathrm{A}, \mathrm{F}$ & 0.609 \\
\hline \multicolumn{10}{|c|}{ Charadriiformes Burhinidae (1) } \\
\hline Great Thick-knee & $\begin{array}{l}\text { Esacus recurvirostris } \\
\text { (Cuvier, 1829) }\end{array}$ & $\mathrm{R}$ & C & NT & - & IV & $\downarrow$ & $\mathrm{RB}, \mathrm{M}$ & 0.38 \\
\hline \multicolumn{10}{|l|}{ Charadriidae (2) } \\
\hline Little Ringed Plover & $\begin{array}{l}\text { Charadrius dubius } \\
\text { (Scopoli, 1786) }\end{array}$ & $\mathrm{R}$ & $\mathrm{O}$ & LC & - & IV & $\rightarrow$ & $\mathrm{RB}, \mathrm{M}$ & 1.842 \\
\hline $\begin{array}{l}\text { Red-wattled } \\
\text { Lapwing }\end{array}$ & $\begin{array}{l}\text { Vanellus indicus } \\
\text { (Boddaert, 1783) }\end{array}$ & $\mathrm{R}$ & $\mathrm{O}$ & LC & - & IV & $?$ & $\mathrm{RB}, \mathrm{M}, \mathrm{A}$ & 1.837 \\
\hline \multicolumn{10}{|l|}{ Jacanidae (1) } \\
\hline $\begin{array}{l}\text { Bronze-winged } \\
\text { Jacana }\end{array}$ & $\begin{array}{l}\text { Metopidius indicus } \\
\text { (Latham, 1790) }\end{array}$ & $\mathrm{R}$ & $\mathrm{O}$ & LC & - & IV & $?$ & A & 0.268 \\
\hline \multicolumn{10}{|l|}{ Laridae (3) } \\
\hline Black-bellied Tern & $\begin{array}{l}\text { Sterna acuticauda } \\
\text { (Gray, 1832) }\end{array}$ & $\mathrm{R}$ & C & EN & - & IV & $\downarrow$ & RB & 0.17 \\
\hline Brown-headed Gull & $\begin{array}{l}\text { Larus brunnicephalus } \\
\text { (Jerdon, 1840) }\end{array}$ & $\mathrm{R}$ & $\mathrm{C}$ & LC & - & IV & $\rightarrow$ & $\mathrm{RC}$ & 0.935 \\
\hline River Tern & $\begin{array}{l}\text { Sterna aurantia (Gray, } \\
\text { 1831) }\end{array}$ & $\mathrm{R}$ & C & VU & - & IV & $\downarrow$ & M & 0.533 \\
\hline \multicolumn{10}{|l|}{ Recurvirostridae (1) } \\
\hline Black-winged Stilt & $\begin{array}{l}\text { Himantopus } \\
\text { himantopus (Linnaeus, } \\
1758 \text { ) }\end{array}$ & WV & C & LC & - & IV & $\uparrow$ & $\mathrm{RB}, \mathrm{M}$ & 0.592 \\
\hline \multicolumn{10}{|l|}{ Scolopacidae (1) } \\
\hline Common Sandpiper & $\begin{array}{l}\text { Actitis hypoleucos } \\
\text { (Linnaeus, 1758) }\end{array}$ & WV & C & LC & - & IV & $\downarrow$ & $\mathrm{RB}, \mathrm{M}$ & 0.632 \\
\hline \multicolumn{10}{|c|}{ Columbiformes Columbidae (4) } \\
\hline $\begin{array}{l}\text { Eurasian Collared } \\
\text { Dove }\end{array}$ & $\begin{array}{l}\text { Streptopelia decaocto } \\
\text { (Frivaldszky, 1838) }\end{array}$ & $\mathrm{R}$ & G & LC & - & IV & $\uparrow$ & $\mathrm{RB}, \mathrm{A}, \mathrm{F}$ & 0.661 \\
\hline Red Collared Dove & $\begin{array}{l}\text { Streptopelia } \\
\text { tranquebarica } \\
\text { (Hermann, 1804) }\end{array}$ & $\mathrm{R}$ & G & LC & - & IV & $\downarrow$ & $\mathrm{RB}, \mathrm{A}, \mathrm{F}$ & 0.632 \\
\hline Rock Pigeon & $\begin{array}{l}\text { Columba livia (Gmelin, } \\
\text { 1789) }\end{array}$ & $\mathrm{R}$ & G & LC & - & IV & $\downarrow$ & $\mathrm{RB}, \mathrm{A}, \mathrm{F}$ & 0.555 \\
\hline
\end{tabular}


Table 1. Checklist and encounter rate of avifauna recorded in riverine areas of Ayodhya district, Uttar Pradesh, India

\begin{tabular}{|c|c|c|c|c|c|c|c|c|c|}
\hline \multirow{2}{*}{$\begin{array}{l}\text { Order/Family/ } \\
\text { Common name }\end{array}$} & \multirow[t]{2}{*}{ Scientific name } & \multirow{2}{*}{$\begin{array}{l}\text { Residential } \\
\text { status }\end{array}$} & \multirow{2}{*}{$\begin{array}{l}\text { Feeding } \\
\text { guild }\end{array}$} & \multicolumn{4}{|c|}{ Conservation status } & \multirow[t]{2}{*}{ Habitat (s) } & \multirow{2}{*}{$\begin{array}{l}\text { Encounter } \\
\text { rate }\end{array}$} \\
\hline & & & & $\begin{array}{c}\text { IUCN list } \\
(2021)\end{array}$ & $\begin{array}{l}\text { CITES } \\
(2012) \\
\end{array}$ & $\begin{array}{l}\text { IWPA } \\
(1972)\end{array}$ & $\begin{array}{l}\text { Global } \\
\text { status }\end{array}$ & & \\
\hline $\begin{array}{l}\text { Yellow-footed } \\
\text { Green-pigeon }\end{array}$ & $\begin{array}{l}\text { Treron phoenicopterus } \\
\text { (Latham, 1790) }\end{array}$ & $\mathrm{R}$ & $\mathrm{F}$ & LC & - & IV & $\uparrow$ & $\mathrm{F}$ & 0.84 \\
\hline \multicolumn{10}{|c|}{ Coraciiformes Alcedinidae (2) } \\
\hline Pied Kingfisher & $\begin{array}{l}\text { Ceryle rudis } \\
\text { (Linnaeus, 1758) }\end{array}$ & $\mathrm{R}$ & $\mathrm{C}$ & LC & - & IV & $?$ & $\mathrm{RB}, \mathrm{M}$ & 0.679 \\
\hline $\begin{array}{l}\text { White-throated } \\
\text { Kingfisher }\end{array}$ & $\begin{array}{l}\text { Halcyon smyrnensis } \\
\text { (Linnaeus, 1758) }\end{array}$ & $\mathrm{R}$ & C & LC & - & IV & $\uparrow$ & $\mathrm{RB}, \mathrm{M}, \mathrm{A}$ & 0.546 \\
\hline \multicolumn{10}{|l|}{ Coraciidae (1) } \\
\hline Indian Roller & $\begin{array}{l}\text { Coracias benghalensis } \\
\text { (Linnaeus, 1758) }\end{array}$ & $\mathrm{R}$ & C & LC & - & IV & $\uparrow$ & $A, F$ & 1.177 \\
\hline \multicolumn{10}{|l|}{ Meropidae (2) } \\
\hline $\begin{array}{l}\text { Blue-tailed Bee- } \\
\text { eater }\end{array}$ & $\begin{array}{l}\text { Merops philippinus } \\
\text { (Linnaeus, 1767) }\end{array}$ & SV & 1 & LC & - & IV & $\rightarrow$ & $A, F$ & 0.054 \\
\hline Green Bee-eater & $\begin{array}{l}\text { Merops orientalis } \\
\text { (Latham, 1801) }\end{array}$ & $\mathrm{R}$ & I & LC & - & IV & $\uparrow$ & $A, F$ & 0.764 \\
\hline \multicolumn{10}{|c|}{ Galliformes Phasianidae (1) } \\
\hline Indian Peafowl & $\begin{array}{l}\text { Pavo cristatus } \\
\text { (Linnaeus, 1758) }\end{array}$ & $\mathrm{R}$ & $\mathrm{O}$ & LC & III & I & $\rightarrow$ & $A, F$ & 0.872 \\
\hline \multicolumn{10}{|c|}{ Gruiformes Gruidae (1) } \\
\hline Sarus Crane & $\begin{array}{l}\text { Antigone antigone } \\
\text { (Linnaeus, 1758) }\end{array}$ & $\mathrm{R}$ & $\mathrm{O}$ & VU & - & IV & $\downarrow$ & $\mathrm{RB}, \mathrm{M}, \mathrm{A}$ & 0.39 \\
\hline \multicolumn{10}{|l|}{ Rallidae (2) } \\
\hline Common Moorhen & $\begin{array}{l}\text { Gallinula chloropus } \\
\text { (Linnaeus, 1758) }\end{array}$ & $\mathrm{R}$ & $\mathrm{O}$ & LC & - & IV & $\rightarrow$ & $\mathrm{RC}, \mathrm{M}$ & 0.924 \\
\hline $\begin{array}{l}\text { White-breasted } \\
\text { Waterhen }\end{array}$ & $\begin{array}{l}\text { Amaurornis } \\
\text { phoenicurus (Pennant, } \\
1769 \text { ) }\end{array}$ & $\mathrm{R}$ & $\mathrm{O}$ & LC & - & IV & $?$ & $\begin{array}{l}\mathrm{RC}, \mathrm{RB}, \mathrm{M}, \\
\mathrm{A}\end{array}$ & 0.962 \\
\hline \multicolumn{10}{|c|}{ Passeriformes Alaudidae (1) } \\
\hline Sand Lark & $\begin{array}{l}\text { Alaudala raytal (Blyth, } \\
\text { 1845) }\end{array}$ & $\mathrm{R}$ & $\mathrm{O}$ & LC & - & IV & $\rightarrow$ & A & 0.446 \\
\hline \multicolumn{10}{|l|}{ Cisticolidae (2) } \\
\hline Ashy Prinia & $\begin{array}{l}\text { Prinia socialis (Sykes, } \\
\text { 1832) }\end{array}$ & $\mathrm{R}$ & 1 & LC & - & IV & $\rightarrow$ & $A, F$ & 0.592 \\
\hline Plain Prinia & $\begin{array}{l}\text { Prinia inornata (Sykes, } \\
\text { 1832) }\end{array}$ & $\mathrm{R}$ & I & LC & - & IV & $\rightarrow$ & $A, F$ & 0.857 \\
\hline \multicolumn{10}{|l|}{ Corvidae (3) } \\
\hline House Crow & $\begin{array}{l}\text { Corvus splendens } \\
\text { (Vieillot, 1817) }\end{array}$ & $\mathrm{R}$ & $\mathrm{O}$ & LC & - & V & $\rightarrow$ & $\mathrm{RB}, \mathrm{M}, \mathrm{A}, \mathrm{F}$ & 1.787 \\
\hline Large-billed Crow & $\begin{array}{l}\text { Corvus } \\
\text { macrorhynchos } \\
\text { (Wagler, 1827) }\end{array}$ & $\mathrm{R}$ & $\mathrm{O}$ & LC & - & IV & $\rightarrow$ & $\mathrm{RB}, \mathrm{M}, \mathrm{A}, \mathrm{F}$ & 2.125 \\
\hline Rufous Treepie & $\begin{array}{l}\text { Dendrocitta } \\
\text { vagabunda (Latham, } \\
\text { 1790) }\end{array}$ & $\mathrm{R}$ & $\mathrm{O}$ & LC & - & IV & $\downarrow$ & $A, F$ & 0.549 \\
\hline \multicolumn{10}{|l|}{ Dicruridae (1) } \\
\hline Black Drongo & $\begin{array}{l}\text { Dicrurus macrocercus } \\
\text { (Vieillot, 1817) }\end{array}$ & $\mathrm{R}$ & C & LC & - & IV & $?$ & $A, F$ & 0.36 \\
\hline \multicolumn{10}{|l|}{ Estrildidae (2) } \\
\hline Indian Silverbill & $\begin{array}{l}\text { Euodice malabarica } \\
\text { (Linnaeus, 1758) }\end{array}$ & $\mathrm{R}$ & G & LC & - & IV & $\rightarrow$ & A & 2.685 \\
\hline $\begin{array}{l}\text { Scaly-breasted } \\
\text { Munia }\end{array}$ & $\begin{array}{l}\text { Lonchura punctulata } \\
\text { (Linaeus, 1758) }\end{array}$ & $\mathrm{R}$ & $\mathrm{O}$ & LC & - & IV & $\rightarrow$ & A & 0.979 \\
\hline
\end{tabular}


Table 1. Checklist and encounter rate of avifauna recorded in riverine areas of Ayodhya district, Uttar Pradesh, India

\begin{tabular}{|c|c|c|c|c|c|c|c|c|c|}
\hline \multirow{2}{*}{$\begin{array}{l}\text { Order/Family/ } \\
\text { Common name }\end{array}$} & \multirow[t]{2}{*}{ Scientific name } & \multirow{2}{*}{$\begin{array}{l}\text { Residential } \\
\text { status }\end{array}$} & \multirow{2}{*}{$\begin{array}{l}\text { Feeding } \\
\text { guild }\end{array}$} & \multicolumn{4}{|c|}{ Conservation status } & \multirow[t]{2}{*}{ Habitat (s) } & \multirow{2}{*}{$\begin{array}{l}\text { Encounter } \\
\text { rate }\end{array}$} \\
\hline & & & & $\begin{array}{l}\text { IUCN list } \\
(2021)\end{array}$ & $\begin{array}{l}\text { CITES } \\
(2012)\end{array}$ & $\begin{array}{l}\text { IWPA } \\
(1972)\end{array}$ & $\begin{array}{l}\text { Global } \\
\text { status }\end{array}$ & & \\
\hline \multicolumn{10}{|l|}{ Hirundinidae (1) } \\
\hline Barn Swallow & $\begin{array}{l}\text { Hirundo rustica } \\
\text { (Linnaeus, 1758) }\end{array}$ & WV & 1 & LC & - & IV & $\downarrow$ & $\mathrm{RB}, \mathrm{A}, \mathrm{M}$ & 0.839 \\
\hline \multicolumn{10}{|l|}{ Laniidae (1) } \\
\hline Long-tailed Shrike & $\begin{array}{l}\text { Lanius schach } \\
\text { (Linnaeus, 1758) }\end{array}$ & $\mathrm{R}$ & $\mathrm{C}$ & LC & - & IV & $?$ & $\mathrm{M}, \mathrm{A}$ & 0.644 \\
\hline \multicolumn{10}{|l|}{ Leiothrichidae (1) } \\
\hline Common Babbler & $\begin{array}{l}\text { Argya caudata } \\
\text { (Dumont, 1823) }\end{array}$ & $\mathrm{R}$ & $\mathrm{O}$ & LC & - & IV & $\rightarrow$ & $\mathrm{M}, \mathrm{A}, \mathrm{F}$ & 2.113 \\
\hline \multicolumn{10}{|l|}{ Motacillidae (4) } \\
\hline Citrine Wagtail & $\begin{array}{l}\text { Motacilla citreola } \\
\text { (Pallas, 1776) }\end{array}$ & WV & 1 & LC & - & IV & $\uparrow$ & $\mathrm{RB}, \mathrm{M}$ & 1.499 \\
\hline Grey Wagtail & $\begin{array}{l}\text { Motacilla cinerea } \\
\text { (Tunstall, 1771) }\end{array}$ & WV & 1 & LC & - & IV & $\rightarrow$ & $\mathrm{RB}, \mathrm{M}$ & 0.725 \\
\hline White Wagtail & $\begin{array}{l}\text { Motacilla alba } \\
\text { (Linnaeus, 1758) }\end{array}$ & WV & 1 & LC & - & IV & $\rightarrow$ & RB & 1.482 \\
\hline $\begin{array}{l}\text { White-browed } \\
\text { Wagtail }\end{array}$ & $\begin{array}{l}\text { Motacilla } \\
\text { maderaspatensis } \\
\text { (Gmelin, 1789) }\end{array}$ & $\mathrm{R}$ & 1 & LC & - & IV & $\rightarrow$ & $\mathrm{RB}, \mathrm{M}$ & 1.161 \\
\hline \multicolumn{10}{|l|}{ Muscicapidae (2) } \\
\hline Indian Robin & $\begin{array}{l}\text { Copsychus fulicatus } \\
\text { (Linnaeus, 1766) }\end{array}$ & $\mathrm{R}$ & C & LC & - & IV & $\rightarrow$ & $A, F$ & 1.766 \\
\hline $\begin{array}{l}\text { Oriental Magpie } \\
\text { Robin }\end{array}$ & $\begin{array}{l}\text { Copsychus saularis } \\
\text { (Linnaeus, 1758) }\end{array}$ & $\mathrm{R}$ & C & LC & - & IV & $\rightarrow$ & $M, A, F$ & 0.754 \\
\hline \multicolumn{10}{|l|}{ Passeridae (1) } \\
\hline House Sparrow & $\begin{array}{l}\text { Passer domesticus } \\
\text { (Linnaeus, 1758) }\end{array}$ & $\mathrm{R}$ & $\mathrm{O}$ & LC & - & IV & $\downarrow$ & $\mathrm{RB}, \mathrm{A}, \mathrm{F}$ & 2.44 \\
\hline \multicolumn{10}{|l|}{ Pycnonotidae (1) } \\
\hline Red-vented Bulbul & $\begin{array}{l}\text { Pycnonotus cafer } \\
\text { (Linnaeus, 1766) }\end{array}$ & $\mathrm{R}$ & $\mathrm{O}$ & LC & - & IV & $\uparrow$ & $\mathrm{M}, \mathrm{A}, \mathrm{F}$ & 2.471 \\
\hline \multicolumn{10}{|l|}{ Sturnidae (4) } \\
\hline Asian Pied Starling & $\begin{array}{l}\text { Gracupica contra } \\
\text { (Linnaeus, 1758) }\end{array}$ & $\mathrm{R}$ & $\mathrm{O}$ & LC & - & IV & $\uparrow$ & $R B, A, F$ & 1.477 \\
\hline Bank Myna & $\begin{array}{l}\text { Acridotheres } \\
\text { ginginianus (Latham, } \\
\text { 1790) }\end{array}$ & $\mathrm{R}$ & $\mathrm{O}$ & LC & - & IV & $\uparrow$ & $\mathrm{RB}, \mathrm{M}$ & 0.931 \\
\hline Brahminy Starling & $\begin{array}{l}\text { Sturnia pagodarum } \\
\text { (Gmelin, 1789) }\end{array}$ & $\mathrm{R}$ & $\mathrm{O}$ & LC & - & IV & $?$ & $A, F$ & 0.536 \\
\hline Common Myna & $\begin{array}{l}\text { Acridotheres tristis } \\
\text { (Linnaeus, 1766) }\end{array}$ & $\mathrm{R}$ & $\mathrm{O}$ & LC & - & IV & $\uparrow$ & $\mathrm{M}, \mathrm{A}, \mathrm{F}$ & 1.187 \\
\hline \multicolumn{10}{|c|}{ Pelecaniformes Anhingidae (1) } \\
\hline Oriental Darter & $\begin{array}{l}\text { Anhinga melanogaster } \\
\text { (Pennant, 1769) }\end{array}$ & WV & $\mathrm{O}$ & NT & - & IV & $\downarrow$ & $\mathrm{RB}, \mathrm{M}$ & 0.695 \\
\hline \multicolumn{10}{|l|}{ Ardeidae (6) } \\
\hline Cattle Egret & $\begin{array}{l}\text { Bubulcus ibis } \\
\text { (Linnaeus, 1758) }\end{array}$ & $\mathrm{R}$ & $\mathrm{C}$ & LC & - & IV & $\uparrow$ & $\mathrm{RB}, \mathrm{M}, \mathrm{A}$ & 2.12 \\
\hline Great Egret & $\begin{array}{l}\text { Ardea alba (Linnaeus, } \\
\text { 1758) }\end{array}$ & $\mathrm{R}$ & $\mathrm{C}$ & LC & - & IV & $?$ & $\mathrm{M}, \mathrm{A}$ & 0.333 \\
\hline Grey Heron & $\begin{array}{l}\text { Ardea cinerea } \\
\text { (Linnaeus, 1758) }\end{array}$ & WV & $\mathrm{C}$ & LC & - & IV & $?$ & $\mathrm{RB}, \mathrm{M}$ & 0.453 \\
\hline Indian Pond Heron & $\begin{array}{l}\text { Ardeola grayii (Sykes, } \\
\text { 1832) }\end{array}$ & $\mathrm{R}$ & $\mathrm{C}$ & LC & - & IV & $?$ & $\mathrm{RB}, \mathrm{M}$ & 1.392 \\
\hline Intermediate Egret & $\begin{array}{l}\text { Ardea intermedia } \\
\text { (Wagler, 1829) }\end{array}$ & $\mathrm{R}$ & $\mathrm{C}$ & LC & - & IV & $\downarrow$ & $\mathrm{RB}, \mathrm{M}$ & 2.086 \\
\hline Little Egret & $\begin{array}{l}\text { Egretta garzetta } \\
\text { (Linnaeus, 1766) }\end{array}$ & $\mathrm{R}$ & C & LC & - & IV & $\uparrow$ & $\mathrm{RB}, \mathrm{M}, \mathrm{A}$ & 2.595 \\
\hline
\end{tabular}


Table 1. Checklist and encounter rate of avifauna recorded in riverine areas of Ayodhya district, Uttar Pradesh, India

\begin{tabular}{|c|c|c|c|c|c|c|c|c|c|}
\hline \multirow{2}{*}{$\begin{array}{l}\text { Order/Family/ } \\
\text { Common name }\end{array}$} & \multirow[t]{2}{*}{ Scientific name } & \multirow{2}{*}{$\begin{array}{l}\text { Residential } \\
\text { status }\end{array}$} & \multirow{2}{*}{$\begin{array}{l}\text { Feeding } \\
\text { guild }\end{array}$} & \multicolumn{4}{|c|}{ Conservation status } & \multirow[t]{2}{*}{ Habitat (s) } & \multirow{2}{*}{$\begin{array}{l}\text { Encounter } \\
\text { rate }\end{array}$} \\
\hline & & & & $\begin{array}{l}\text { IUCN list } \\
(2021)\end{array}$ & $\begin{array}{l}\text { CITES } \\
(2012)\end{array}$ & $\begin{array}{l}\text { IWPA } \\
(1972)\end{array}$ & $\begin{array}{l}\text { Global } \\
\text { status }\end{array}$ & & \\
\hline \multicolumn{10}{|l|}{ Ciconiidae (3) } \\
\hline Asian Openbill & $\begin{array}{l}\text { Anastomus oscitans } \\
\text { (Boddaert, 1783) }\end{array}$ & $\mathrm{R}$ & C & LC & - & IV & $?$ & $\mathrm{RC}$ & 1.77 \\
\hline Painted Stork & $\begin{array}{l}\text { Mycteria leucocephala } \\
\text { (Pennant, 1769) }\end{array}$ & WV & C & NT & - & IV & $\downarrow$ & $\mathrm{RC}, \mathrm{RB}, \mathrm{M}$ & 0.582 \\
\hline $\begin{array}{l}\text { Woolly-necked } \\
\text { Stork }\end{array}$ & $\begin{array}{l}\text { Ciconia episcopus } \\
\text { (Boddaert, 1783) }\end{array}$ & $\mathrm{R}$ & C & NT & - & IV & $\downarrow$ & A & 1.126 \\
\hline \multicolumn{10}{|c|}{ Phalacrocoracidae (1) } \\
\hline Little Cormorant & $\begin{array}{l}\text { Microcarbo niger } \\
\text { (Vieillot, 1817) }\end{array}$ & $\mathrm{R}$ & $\mathrm{C}$ & LC & - & IV & $?$ & RB & 2.569 \\
\hline \multicolumn{10}{|c|}{ Psittaciformes Psittaculidae (1) } \\
\hline $\begin{array}{l}\text { Rose-ringed } \\
\text { Parakeet }\end{array}$ & $\begin{array}{l}\text { Psittacula krameri } \\
\text { (Scopoli, 1769) }\end{array}$ & $\mathrm{R}$ & $\mathrm{F}$ & LC & - & IV & $\uparrow$ & $A, F$ & 0.691 \\
\hline \multicolumn{10}{|c|}{ Strigiformes Strigidae (2) } \\
\hline Jungle Owlet & $\begin{array}{l}\text { Glaucidium radiatum } \\
\text { (Tickell, 1833) }\end{array}$ & $\mathrm{R}$ & C & LC & - & IV & $\rightarrow$ & $A, F$ & 0.198 \\
\hline Spotted Owlet & $\begin{array}{l}\text { Athene brama } \\
\text { (Temminck, 1821) }\end{array}$ & $\mathrm{R}$ & C & LC & II & IV & $\rightarrow$ & $A, F$ & 0.316 \\
\hline
\end{tabular}

IUCN: International Union for Conservation of Nature and Natural Resources; CITES: Convention on International Trade in Endangered Species of Wild Fauna and Flora; IPWA: Indian Wildlife Protection Act; R: Resident, WV: Winter Visitor, SV: Summer Visitor; C: Carnivorous; O: Omnivorous; I: Insectivorous; F: Frugivorous; G: Granivorous; LC: Least Concern; EN: Endangered; VU: Vulnerable; NT: Near Threatened; CITES II: Schedule-II species of CITES are the ones that are not necessarily threatened now with extinction but may become so unless trade is closely controlled; IWPA I: Schedule - I species of IWPA (high priority species); IV: Schedule - IV species of IWPA (relatively low priority species); ?: Unknown; —: Stable; $\uparrow:$ Increasing; $\downarrow$ : Decreasing; RB: River Bank; A: Agricultural field, F: Forested area, M: Marshland; RC: River Course

the Central Asian Flyway and thus might be supporting high diversity of migratory birds. The resident as well as migratory birds preferring this area indicates the good habitat quality of the riverscape. The birds recorded in this study were observed to be well distributed among different habitats of the riverscapes suggesting that not only the river but also the banks are equally rich in biodiversity and provide the basic requirements of the birds.

According to the feeding guilds, the 68 bird species recorded were classified into five foraging guilds. The maximum number of species recorded were Carnivores (29 species, $43 \%$ ), followed by omnivores ( 24 species, $35 \%$ ) and the least number of species were frugivores (2 species, $3 \%$ )

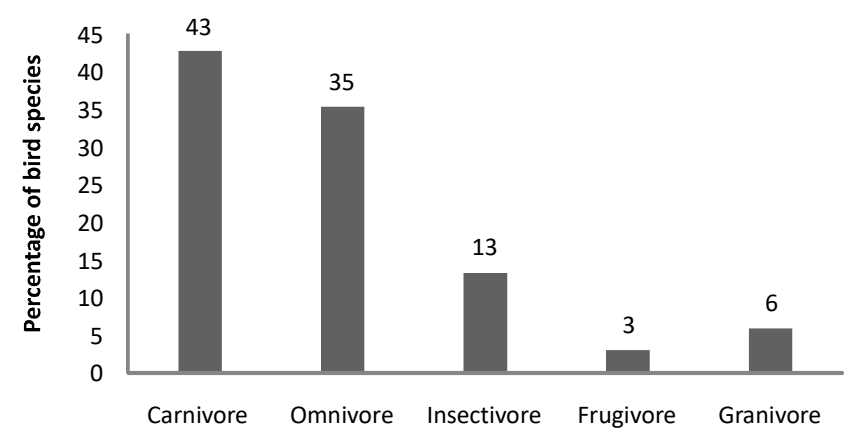

Fig. 4. Foraging guild-based classification of bird species in riverscapes of Ayodhya district, Uttar Pradesh
(Fig. 4). This result again suggests that the area provides the birds with diverse food items. According to the IUCN Red List (2021), out of the 68 bird species recorded, two species (3\%) were 'Endangered', three species (4\%) were 'Vulnerable', four species $(6 \%)$ were 'Near Threatened' and the rest 59 species (87\%) were 'Least Concern' (Table 1). Moreover, four species were included in the Appendix-II and one species

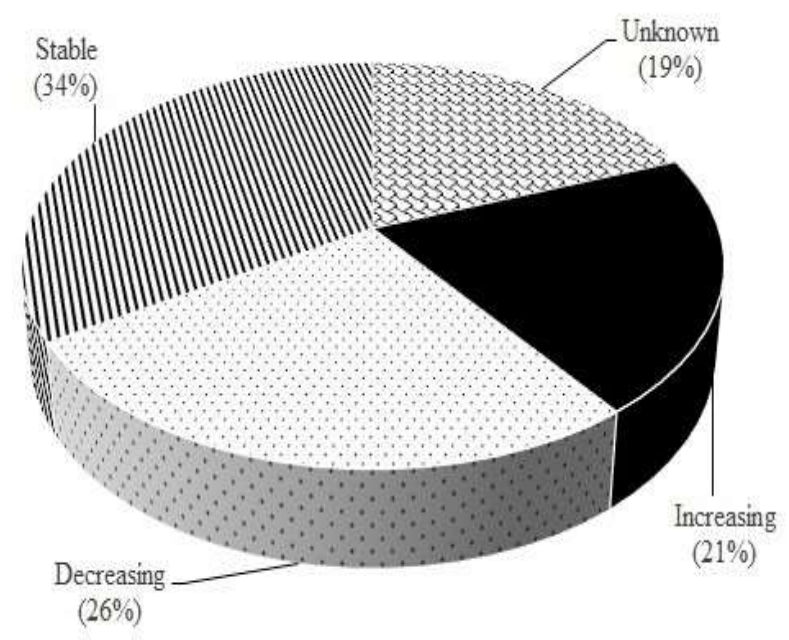

Fig. 5. Global population status of bird species recorded in riverscapes of Ayodhya district, Uttar Pradesh 
Table 2. Relative diversity (Rdi) of various avian families in riverscapes of Ayodhya district, Uttar Pradesh

\begin{tabular}{|c|c|c|}
\hline Avian family & Number of species recorded & Rdi value \\
\hline Ardeidae & 6 & 8.82 \\
\hline Accipitridae & 4 & 5.88 \\
\hline Columbidae & 4 & 5.88 \\
\hline Motacillidae & 4 & 5.88 \\
\hline Sturnidae & 4 & 5.88 \\
\hline Anatidae & 3 & 4.41 \\
\hline Ciconiidae & 3 & 4.41 \\
\hline Corvidae & 3 & 4.41 \\
\hline Laridae & 3 & 4.41 \\
\hline Alcedinidae & 2 & 2.94 \\
\hline Charadriidae & 2 & 2.94 \\
\hline Cisticolidae & 2 & 2.94 \\
\hline Estrildidae & 2 & 2.94 \\
\hline Meropidae & 2 & 2.94 \\
\hline Muscicapidae & 2 & 2.94 \\
\hline Rallidae & 2 & 2.94 \\
\hline Strigidae & 2 & 2.94 \\
\hline Alaudidae & 1 & 1.47 \\
\hline Anhingidae & 1 & 1.47 \\
\hline Burhinidae & 1 & 1.47 \\
\hline Coraciidae & 1 & 1.47 \\
\hline Dicruridae & 1 & 1.47 \\
\hline Gruidae & 1 & 1.47 \\
\hline Hirundinidae & 1 & 1.47 \\
\hline Jacanidae & 1 & 1.47 \\
\hline Laniidae & 1 & 1.47 \\
\hline Leiothrichidae & 1 & 1.47 \\
\hline Passeridae & 1 & 1.47 \\
\hline Phalacrocoracidae & 1 & 1.47 \\
\hline Phasianidae & 1 & 1.47 \\
\hline Psittaculidae & 1 & 1.47 \\
\hline Pycnonotidae & 1 & 1.47 \\
\hline Recurvirostridae & 1 & 1.47 \\
\hline Scolopacidae & 1 & 1.47 \\
\hline Upupidae & 1 & 1.47 \\
\hline
\end{tabular}

was included in Appendix-III of CITES (CITES 2012) (Table 1). Five species came under Schedule I of the Indian Wildlife (Protection) Act (1972) (Table 1). In addition to this, the riverscape supported 18 species $(26 \%)$ of birds having a declining population trend globally (Fig. 5). This work thus, highlights the conservation value of the riverscape of Saryu river.

\section{CONCLUSION}

The high species richness and presence of species of conservation importance along the riverscape of Saryu river indicates the ecological importance of the area. This result also lays emphasis on the need for proper management and conservation of this river and its surrounding landscapes. But threats like intensification and expansion of agriculture and developmental activities loom over this biodiversity rich area. Therefore, there is an urgent need to assess the level of biodiversity and health of this ecosystem all along its stretch. Future in-depth studies must be conducted in this riverscape using a multi-organismal approach. In addition to this, awareness programs at local level are necessary.

\section{ACKNOWLEDGEMENTS}

The author is thankful to the Dean, College of Horticulture and Forestry, ANDUAT, and to the local people for permitting to carry out the field research.

\section{REFERENCES}

Adel MM 2001. Effect on water resources from upstream water diversion in the Ganges Basin. Journal of Environmental Quality 30: 356-368.

Anonymous 2021. Krishi Vigyan Kendra, Ayodhya. https://ayodhya.kvk4.in/ Accessed on $1^{\text {st }}$ September 2021.

Bashir T, Behera SK, Khan A and Gautam P 2012. An inventory of mammals, birds and reptiles along a section of the river and banks of upper Ganges, India. Journal of Threatened Taxa 4(9): 29002910

Behera SK 1995. Studies on Population Dynamics, Habitat Utilization and Conservation Aspects of for Gangetic Dolphin (Platanista gangetica) in a stretch of Ganga River from Rishekesh to Kanpur. Ph.D. Thesis, School of Studies in Zoology, Jiwaji University, Gawalior, India.

CITES (Convention on International Trade in Endangered Species of Wild Fauna and Flora) 2012. Checklist of Convention on International Trade in Endangered Species of Wild Fauna and Flora. CITES, Geneva, Switzerland. http://www.cites.org. Accessed on $4^{\text {th }}$ September 2021.

Datta A and Goyal SP 2008. Responses of diurnal tree squirrels to selective logging in western Arunachal Pradesh. Current Science 95(7): 895-902

Dhar O N and Nandargi S 2002. Flood study of the Himalayan tributaries of the Ganga river. Meteorological Applications 9: 63-68

Dudgeon D, Arthington A, Gessner M, Kawabata Z, Knowler D, Leveque $C$, Naiman $R$, Prieur-Richard A, Soto D, Stiassny $M$ and Sullivan C 2006. Freshwater biodiversity: importance, threats, status and conservation challenges. Biological Review 81: 163182.

Gregory RD, Gibbons DW and Donald PF 2004. Bird census and survey techniques, pp. 17-56. In: Sutherland W J, Newton I and Green R (eds). Bird ecology and conservation: A Handbook of Techniques. New York, Oxford University Press Inc., London, U.K.

Grimmett R, Inskipp C and Inskipp T 2011. Birds of the Indian Subcontinent. Oxford University Press and Christopher Helm, London, U.K. p. 480.

Hawksworth D and Kalin-Arroyo M 1995. Magnitude and distribution of biodiversity, pp. 107-191. In: Heywood V H (ed). Global biodiversity Assessment, Cambridge University Press, Cambridge, U.K. 
IUCN (International Union for Conservation of Nature) 2021. The IUCN Red List of threatened species, v. 2017.1. BirdLife International, Gland, Switzerland. http://www.iucnredlist.org. Accessed on $4^{\text {th }}$ September 2021.

IWPA (Indian Wildlife Protection Act) 1972. The Indian Wildlife (Protection) Act, 1972 (as amended up to 1993). Ministry of Environment, Forest and Climate Change, Govt. of India, Delhi. http://www.envfor.nic.in/legis/wildlife/wildlife1.html Accessed on $4^{\text {th }}$ September 2021.

Newby E 1998. Slowly Down The Ganges. Lonely Planet Publications, pp. 23-25.

Praveen J, Jayapal R and Pittie A 2016. A checklist of the birds of India. Indian Birds 11(5\&6): 113-172A.

Praveen J, Jayapal R and Pittie A 2020. Taxonomic updates to the checklists of birds of India, and the South Asian region. Indian Birds 16(1): 12-19.

Revenga Cl, Campbell R, Abell de Villiers P and Bryer M 2005.
Prospecting for monitoring freshwater ecosystems towards the 2010 targets. Philosophical Transactions of the Royal Society B. Biological Sciences 360: 397-413.

Singh H, Raghuvanshi D, Pandey R, Yadav A, Tripathi B, Kumar P and Shukla BN 2016. Assessment of seven heavy metals in water of the river Ghaghara, a major tributary of the Ganga in Northern India. Advances in Applied Science Research 7(5): 34-45.

Sinha A, Hariharan H, Adhikari B and Krishnamurthy R 2019. Bird diversity along riverine areas in the Bhagirathi Valley, Uttarakhand, India. Biodiversity Data Journal 7: e31588.

Torre-Cuadros MDLAL, Herrando-Perez S and Young KR 2007. Diversity and structure patterns for tropical montane and premontane forests of central Peru, with an assessment of the use of higher-taxon surrogacy. Biodiversity and Conservation 16 2965-2988.

Wiens J 2002. Riverine landscapes: Taking landscape ecology into the water. Freshwater Biology 47: 501-515.

Received 29 September, 2021; Accepted 10 January, 2022 